\title{
An Inexact Inventory Theory-Based Water Resources Distribution Model for Yuecheng Reservoir, China
}

\author{
Meiqin Suo $\mathbb{D}^{1},{ }^{1}$ Fuhui Du, ${ }^{1}$ Yongping $\mathrm{Li}^{2}$ Tengteng Kong, ${ }^{1}$ and Jing Zhang ${ }^{1}$ \\ ${ }^{1}$ School of Water Conservancy and Hydroelectric Power, Hebei University of Engineering, Handan 056038, China \\ ${ }^{2}$ School of Environment, Beijing Normal University, Beijing 100875, China \\ Correspondence should be addressed to Meiqin Suo; suomeiqin@hebeu.edu.cn
}

Received 11 August 2020; Revised 11 September 2020; Accepted 11 October 2020; Published 31 October 2020

Academic Editor: Huiyan Cheng

Copyright (c) 2020 Meiqin Suo et al. This is an open access article distributed under the Creative Commons Attribution License, which permits unrestricted use, distribution, and reproduction in any medium, provided the original work is properly cited.

In this study, an inexact inventory theory-based water resources distribution (IIWRD) method is advanced and applied for solving the problem of water resources distribution from Yuecheng Reservoir to agricultural activities, in the Zhanghe River Basin, China. In the IIWRD model, the techniques of inventory model, inexact two-stage stochastic programming, and interval-fuzzy mathematics programming are integrated. The water diversion problem of Yuecheng Reservoir is handled under multiple uncertainties. Decision alternatives for water resources allocation under different inflow levels with a maximized system benefit and satisfaction degree are provided for water resources management in Yuecheng Reservoir. The results show that the IIWRD model can afford an effective scheme for solving water distribution problems and facilitate specific water diversion of a reservoir for managers under multiple uncertainties and a series of policy scenarios.

\section{Introduction}

The economic development of a region depends heavily on the water distribution of its large reservoirs. However, reservoir water resource management is complex that involves many factors and processes. For example, it is related with the local natural conditions, the adjustable water diversion from upstream in case of drought, the storage capacity and maintaining condition under different inflow levels, the laying of channels/pipes and the loss in the process of water diversion and supply, and different demands of various water users. Each factor/process has many uncertainties, which become more complex depending on the quality of the obtained data [1]. In addition, with the different levels of economic development, water demand will change dynamically with various temporal and spatial scales. Therefore, effectively optimizing the water distribution of a reservoir under uncertainty would be helpful to realize the sustainable development of the region, especially reducing the risk of water shortage under severe drought.

Over the past decades, a number of simulation and optimization methods were developed for reservoir water resources management under uncertainty [2-19]. Among them, inexact two-stage stochastic programming (ITSP) is effective for analyzing policy scenarios, and taking corrective actions after a random event has taken place in order to minimize "penalties" that may appear due to incorrect policy [11]. Meanwhile, ITSP can reflect the random uncertainty quantified as probability density functions (PDFs) and handle uncertain parameters or variables with variable ranges in programming problems by interval numbers. Interval-fuzzy mathematics programming is useful for decision problems of fuzzy goals and constraints with fuzziness by establishing fuzzy sets and fuzzy functions under interval uncertainty [20].

However, many parameters in actual problem have no information of probability distribution and change with the different temporal and spatial scales and may be presented as intervals. In addition, these intervals have no determined boundaries at sometimes, such as the system cost, and its boundary is difficult to ascertain associated with the volatility of the market and the change in supply and demand and thus shown with fuzziness. It is noted that when the range of interval parameter is so wide that the final result of 
the model has a wide interval solution, the manager would feel difficult to make decision by ITSP. According to the past studies [21-24], it is known that the interval-fuzzy mathematics programming (IFMP) could well indicate the fuzzy uncertainty by fuzzy number and membership degree and provides interval solution with a certain satisfaction degree under flexible constraints, to avoid the trouble of making decision resulting from the wide interval solution. Accordingly, multiple uncertainties would be handled by incorporating ITSP and IFMP within a general framework.

On the contrary, in reservoir water resource management system, there is a series of process that needs to be considered besides the above described uncertainties. In particular, when the drought is severe and the reservoir is insufficient, the water diversion would be necessary to satisfy the local water demand. In this case, a scheme of water diversion needs to be established according to the adjustable water diversion from upstream, the local water demand, and the reservoir storage limit, including total diversion quantity, diversion batch size, and period per time. The past models for water diversion of reservoirs mainly focused on the total quantity of water diversion, but few studied the specific diversion batch and period [25-29]. As an economic model, the inventory model can effectively tackle the problem of supply and demand and provide the most economical material purchase batch and period. But the past inventory models rarely studied the planning problems for water resources [30-35]. Since water as a resource has been gradually marketized, the combination of inventory model and other uncertain optimization technologies would undoubtedly make the research studies on the planning of water resources system more rich and perfect and has better applicability.

Therefore, the objective of this study is to develop an inexact inventory theory-based water resources distribution (IIWRD) method through incorporating inventory theory, ITSP, and IFMP within a general framework. The IIWRD method will be helpful for quantitatively analyzing a series of policy scenarios under multiple uncertainties; more importantly, it can afford the specific scheme for water diversion associated with different policy scenarios, including the total water diversion, diversion batch, and period per time. Then, IIWRD is applied to planning water resources distribution of Yuecheng Reservoir that is located in Zhanghe River Basin, China. The results will help to identify desired schemes of water diversion and water allocation under different inflow levels with maximized system benefit and satisfaction degree. Furthermore, the system benefit and total water diversion under different scenarios of inflow levels and unit diversion costs are analyzed to help managers make decision. Comprehensively, three special characteristics of IIWRD make it unique after comparing with the existing methods: (i) it can effectively handle multiple forms of uncertainties, (ii) it is helpful to quantitatively analyzing a series of policy scenarios that are related with various levels of economic penalties, and (iii) it can facilitate specific water diversion of a reservoir under multiple uncertainties.

\section{Water Resources Distribution in Yuecheng Reservoir}

The Yuecheng Reservoir is a superlarge reservoir directly under the administration of the state, located in Ci County of Handan City, Hebei Province, controlling a river basin area of $18.1 \times 10^{3} \mathrm{~km}^{2}$. Its main task is flood control, irrigation, urban water supply, and electricity generation. Its total capacity is $1.3 \times 10^{9} \mathrm{~m}^{3}$ and can partially solve the industrial and living water of Handan City and Anyang City. According to historical statistics, the accumulated water supply of the reservoir was $17.17 \times 10^{9} \mathrm{~m}^{3}$ from 1962 to 2005 , and the total water supply to Hebei Province and Henan Province was $11.73 \times 10^{9} \mathrm{~m}^{3}$ and $5.44 \times 10^{9} \mathrm{~m}^{3}$ [36], respectively. In addition, it can irrigate a farmland of $1.47 \times 10^{5}$ ha through Minyou Canal in Hebei Province and Zhangnan Canal in Henan Province. The irrigated areas of Minyou and Zhangnan refer to 15 counties, with many kinds of crops, but mainly wheat, corn, and cotton.

Recently, due to the rainfall decrease and the building of water diversion channels in the upstream river basin, the incoming runoff of Yuecheng Reservoir is reducing year by year, resulting in serious irrigation water shortage and critical water supply problems, especially in the Zhangnan irrigation area. For example, the average annual water supply of Zhangnan irrigation area from the reservoir was $51.7 \times 10^{6} \mathrm{~m}^{3}$ from 1981 to 1986 , and from 1991 to 1996 , it was $30.5 \times 10^{6} \mathrm{~m}^{3}$, but from 2001 to 2006 , it was only $7.0 \times 10^{6} \mathrm{~m}^{3}$ [37]. Moreover, in order to ensure the safe flood of the reservoir, the water of the reservoir on the level of $132 \mathrm{~m}$ would be leaked before the main flood period, about $85 \times 10^{6} \mathrm{~m}^{3}$. After entering the flood period, because the rainfall in the Zhangwei River Basin is small, the river flow and runoff are few, and the water storage of the reservoir would be not enough. As the important water sources of industry and agriculture for Handan City and Anyang City, Yuecheng Reservoir plays a significant role in the economic life in the two cities and bear a huge pressure of water supply [38].

There are three large reservoirs in the upstream that can divert water to Yuecheng Reservoir, namely, Guanhe, Back bay, and Zhangze. According to the data, from 1960 to 2004, the average annual abandoned water of Zhangze Reservoir was $112 \times 10^{6} \mathrm{~m}^{3}$, the average annual leaked water from Guanhe and Back bay was $23 \times 10^{6} \mathrm{~m}^{3}$ and $37 \times 10^{6} \mathrm{~m}^{3}$. Under this condition, if continuous rainfall occurs in the Zhanghe River Basin and the upstream reservoirs store water fully, it could be considered transferring water from them to Yuecheng Reservoir to relieve the local drought [38]. Since the water allocation of Yuecheng Reservoir to Handan City and Anyang City is generally preferred to meet municipal and industrial use, the agricultural water often appears insufficient, having to take the high cost of groundwater to solve water shortage problems. By calculating, the diversion cost from the upstream reservoirs is much less than the cost of groundwater extraction. Therefore, in order to promote the agricultural development and increase its income in the two cities and make full use of the abandoned water from the 
upstream reservoirs, the problem of water distribution from Yuecheng Reservoir to three main crops (wheat, corn, and cotton) of 15 counties in the two irrigation areas would be studied in this paper (shown in Figure 1). It is noted that the water allocation of Yuecheng Reservoir to municipality and industry in two cities is excluded.

\section{Statement of Problems}

The system of water distribution from Yuecheng Reservoir to agriculture involves many processes and components, and there is a lot of uncertainty between them and each other. For example, the water demand for irrigation of three kinds of crops is related to their crop coefficients, evapotranspiration, and growth periods; thus, it is difficult to get definite values. However, they can be obtained with a range by calculating, which are $[3.12,3.46] \times 10^{3} \mathrm{~m}^{3} / \mathrm{ha}$, $[2.08,2.31] \times 10^{3} \mathrm{~m}^{3} / \mathrm{ha}$, and $[2.08,2.31] \times 10^{3} \mathrm{~m}^{3} / \mathrm{ha}$ [39] for wheat, corn, and cotton, individually. In addition, although some uncertainty can be expressed as interval numbers, their boundaries still show uncertainties, such as the unit cost of water diversion has a relationship with total diversion quantity, draw cost of the upstream reservoirs, and the water loss in the diversion way, all of them are difficult to be estimated. Therefore, the unit cost of water diversion would be expressed as a fuzzy-interval number, being $[24,28,32,36] \mathrm{RMB} / 10^{3} \mathrm{~m}^{3}$ in this study, convenient for analyzing the influence of different diversion costs to total diversion quantity. Moreover, because of the significant changes in the annual precipitation and great differences among the seasonal rainfall in the reservoir, as well as the uncertainty of water supply to municipality and industry in the two cities, the water distribution from the reservoir to agriculture is difficult to determine. At this time, the expected objectives for three crops in each subarea need to be set according to their actual planting quantities and then be corrected on the basis of various possible irrigation levels happened. Thus, an effective comprehensive multiple uncertainties method for water distribution from Yuecheng Reservoir to agriculture is expected.

However, in the system of water distribution of Yuecheng Reservoir, when the drought is so serious that the local water demand cannot be met, it would be necessary to consider water diversion from the upstream. At the moment, the manager needs to consider all the problems happened in the process of water diversion, such as reservoir capacity limits, adjustable water from upstream basin, the water supply cost of the upstream basin, the setup cost for water diversion per time, and the storage cost of the reservoir. In addition, under certain amount of water diversion, if it is too much per time, it will not only bring pressure to the reservoir but also cause the stagnation pressure; if it is too little per time, it will increase the diversion frequency and the setup cost. Therefore, the problems of water diversion from the upstream and water allocation of agriculture under minimized system cost should be handled.

\section{Modelling Formulation}

Before constructing the inexact inventory theory-based water resources distribution (IIWRD) model for Yuecheng Reservoir, methods of inexact two-stage stochastic programming (ITSP), interval-fuzzy mathematics programming (IFMP), and inventory theory need to be introduced. ITSP is effective for analyzing policy scenarios, and taking corrective actions after a random event has taken place in order to minimize "penalties" that may appear due to incorrect policy. In addition, it can deal with uncertainties expressed as discrete intervals and PDFs. A typical ITSP model can be rewritten as follows [11]:

$$
f^{ \pm}=\max C_{T_{1}}^{ \pm} X^{ \pm}-\sum_{h=1}^{v} p_{h} D_{T_{2}}^{ \pm} Y^{ \pm},
$$

subject to

$$
\begin{array}{r}
A_{r_{1}}^{ \pm} X^{ \pm}-A_{r_{2}}^{ \pm} Y^{ \pm} \leq w_{h}^{ \pm}, \quad r_{1}, r_{2} \in M ; M=1,2, \cdots, m_{1} ; \\
h=1,2, \cdots, v, \\
A_{r_{3}}^{ \pm} X^{ \pm}+A_{r_{4}}^{ \pm} Y^{ \pm} \leq B^{ \pm}, \quad r_{3}, r_{4} \in M ; M=1,2, \cdots, m_{2}, \\
x_{j}^{ \pm} \geq 0, x_{j}^{ \pm} \in X^{ \pm}, \quad j=1,2, \cdots, n_{1}, \\
y_{j h}^{ \pm} \geq 0, y_{j h}^{ \pm} \in Y^{ \pm}, \quad j=1,2, \cdots, n_{2} ; h=1,2, \cdots, v,
\end{array}
$$

where $w_{h}^{ \pm}$is the discrete value of an interval random variable with probability level $p_{h}, h=1,2, \ldots, v$ and $\sum_{h=1}^{v} p_{h}=1$ and superscripts - and + are lower and upper bounds of interval parameters, respectively. In addition, $X^{ \pm}$and $Y^{ \pm}$are firstand second-stage decision variables, individually; the righthand side coefficients in equation (1b) are presented as probability distributions. An interval $x^{ \pm}$is defined as a range with known upper and lower bounds but unknown distribution: $x^{ \pm}=\left[x^{-}, x^{+}\right]=\left\{t \in x \mid x^{-} \leq t \leq x^{+}\right\}$, where $x^{-}$and $x^{+}$ are the lower and upper bounds of $x^{ \pm}$, individually [11].

However, in real-world problems, the quality of uncertain information obtained mostly cannot be sufficiently satisfactory to be expressed as probabilities or simply presented as interval values. For example, the lower and upper bounds of interval parameters may be fuzzy in nature, resulting in dual uncertainties. Such complexities in uncertainties cannot be handled by the above ITSP model. It is noted the method of IFMP with mixed fuzzyinterval number (FIN) has advantages in tackling such complexities. In detail, the IFMP can be formulated as follows [20]: 


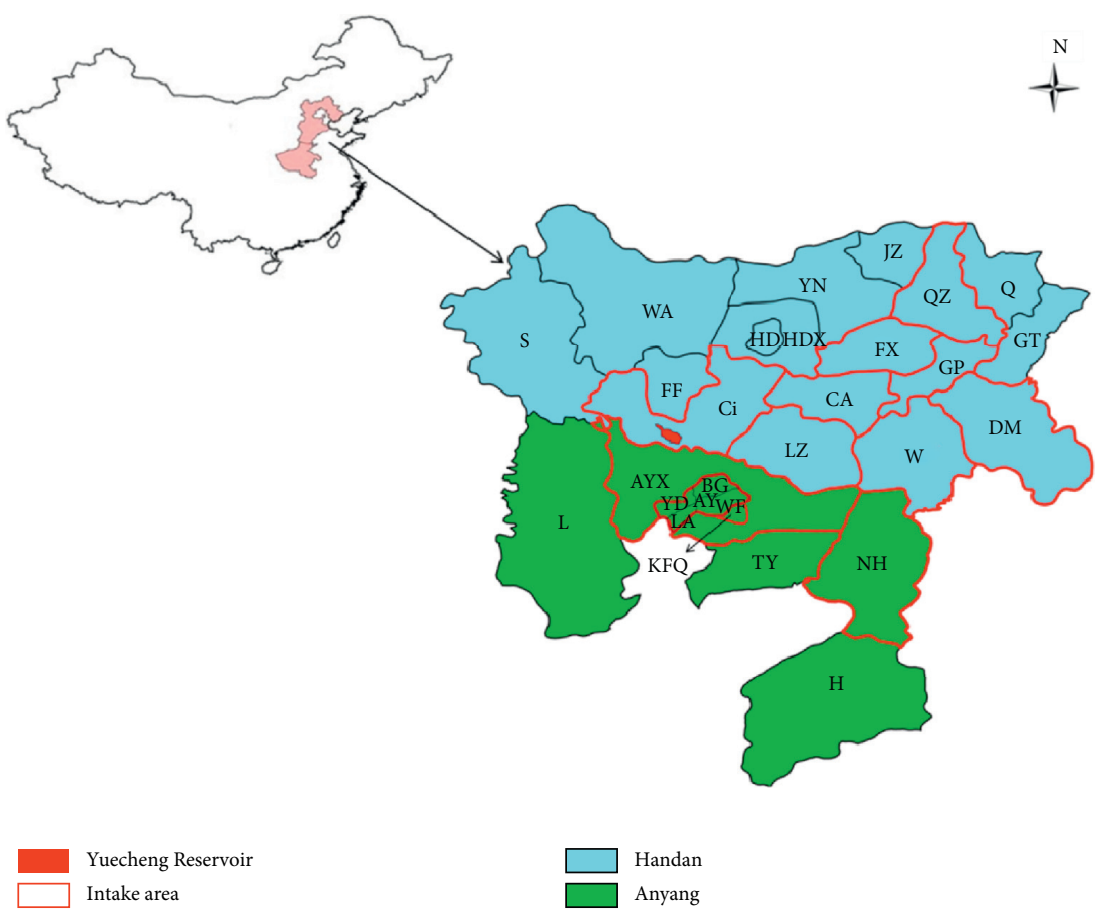

Figure 1: Study area (JZ, Jize County; Q, Qiu County; YN, Yongnian County; GT, Guantao County; WA, Wu'an County; HDX, Handan County; HD, Handan District; S, Shexian; FF, Fengfeng Kuangqu; QZ, Quzhou County; FX, Feixiang County; GP, Guangping County; CA, Chengan County; W, Wei County; Ci, Ci County; LZ, Linzhang County; DM, Daming County; WF, Wenfeng District; BG, Beiguan District; YD, Yindu District; LA, Long'an District; AYX, Anyang County; NH, Neihuang County; KFQ, Kaifaqu; L, Linzhou District; H, Hua County; TY, Tangyin County. QZ, FX, GP, CA, W, Ci, LZ, and DM are irrigated by Minyou Channel and belongs to Handan Municipality, while WF, $\mathrm{BG}, \mathrm{YD}, \mathrm{LA}, \mathrm{AY}, \mathrm{NH}$, and KFQ are irrigated by Zhangnan Channel and belongs to Anyang Municipality).

$$
\operatorname{Max} \lambda^{ \pm}
$$

subject to

$$
\begin{aligned}
C^{ \pm} X^{ \pm} & \geq f^{-}+\lambda^{ \pm}\left(f^{+}-f^{-}\right), \\
A^{ \pm} X^{ \pm} & \leq{\underset{\sim}{B}}^{+}-\lambda^{ \pm} \Delta \underline{\sim}^{ \pm}, \\
X^{ \pm} & \geq 0, \\
0 & \leq \lambda^{ \pm} \leq 1,
\end{aligned}
$$

where $\underline{\sim}^{ \pm}$is a mixed fuzzy-interval number (FIN); let $\Delta \underline{B}^{ \pm}=$ $\underline{\sim}^{+}-\underline{\sim}^{-}=\left[\underline{B}^{+}, \bar{B}^{+}\right]-\left[\underline{B}^{-}, \bar{B}-\right]=\left[\underline{B}^{+}-\bar{B}-\bar{B}^{+}-\underline{B}^{-}\right]$, where $\underline{B}^{-}$ and $\widetilde{\bar{B}}^{-}$are the lower and upper boundaries of the lower interval number, respectively; $\underline{B}^{+}$and $\bar{B}^{+}$are the lower and upper boundaries of the upper interval number, individually. In models $(2 \mathrm{a})-(2 \mathrm{e}), \lambda^{ \pm}$is a control variable. The variable $\lambda^{ \pm}$ represents the degree of satisfaction for the fuzzy objective and/or constraints. When $\lambda^{ \pm}$equals to 1 , it would be related to a solution with the highest possibility of satisfying the constraints/objective under advantageous conditions; inversely, when $\lambda^{ \pm}$equals to zero, it would correspond to a solution that has the lowest possibility of meeting the constraints/objective under demanding conditions.
In the system of water distribution of a reservoir, water diversion would be a prior option when the drought is serious that the local water demand cannot be met. In this case, a series of problems related with water diversion needs to be considered, such as the reservoir capacity, the quantity of water diversion, the diversion period, the cost of water supply, the setup cost for water diversion per time, and the storage cost of the reservoir. Actually, all the problems can be treated as an inventory problem and solved by the economic order quantity (EOQ) model. Based on the assumption of shortages without allowing, a typical EOQ model can be formulated as follows.

Assuming that a material is needed to be produced or purchased, and there is no lead time. The demand is $D$ units per unit time, and the relative costs include $K$ (setup cost for ordering one batch $(\$)$ ), $C$ (unit cost for producing or purchasing each unit (\$/unit)), and $C 1$ (holding cost per unit per unit of time held in inventory (\$/month)). The objective is to determine when and how much to replenish inventory in order to minimize the sum of the produce or purchase costs per unit time [40].

Let the batch be $Q$ and the period be $t$, then $t=Q / D$. Since the period is variable, it is needed to calculate how much the total cost is in a given time (e.g., one month). Under this condition, the sum of the two costs (holding cost 
and ordering cost) described above can be converted into the cost for per month and formulated as follows:

$$
f(Q)=\frac{C_{1} Q}{2}+\frac{K D}{Q}+C D .
$$

By calculating the extreme of $f(Q)$, namely, it can be obtained:

$$
\begin{aligned}
\frac{C_{1}}{2}-\frac{K D}{Q^{2}} & =0, \\
Q^{2} & =\frac{2 K D}{C_{1}}, \\
Q^{*} & =\sqrt{\frac{2 K D}{C_{1}}} .
\end{aligned}
$$

Then, it has $f^{\prime \prime}(Q)=2 K D / Q^{3}$ and $f^{\prime \prime}(Q)>0$ (when $Q>0)$. According to the extremum theorem, $\min f(Q)=$ $f\left(Q^{*}\right)$ when $Q^{*}=\sqrt{2 K D / C_{1}}$. It is the batch formula under the given conditions, usually regarded as the economic order quantity (EOQ) model.

In summary, the models of ITSP, IFMP, and EOQ will be incorporated in this study, which leads to an inexact inventory theory-based water resources distribution (IIWRD) method. Therefore, the IIWRD model can tackle multiple uncertainties within an optimization programming framework by expressing uncertain parameters as discrete intervals, membership functions, probabilistic distributions, and their combinations. Moreover, it can provide corrective measures for the expected objectives against different random events happened. Meanwhile, the inventory scheme also can be gained for handling water diversion problems in water resources management of reservoirs.

To reflect the dynamic variation in water distribution of Yuecheng Reservoir, the water diversion from upstream to
Yuecheng Reservoir and the water allocation from Yuecheng Reservoir to irrigated areas are considered. Because of the limitation of adjustable water diversion from upstream reservoirs, the total amount cannot completely solve the water shortage of the study area, only in the premise of guaranteeing the minimum water supply as much as possible to avoid the water shortage risk. Hence, there may be unirrigated fields under low irrigation level because the use of groundwater is too expensive. In addition, there are some assumptions for the system of water distribution including: (1) water sources for irrigation mainly include surface water and groundwater in this study area. However, since the groundwater is difficult to be used in different places, it would be used in the local area without distributing, while only distributes the surface water; (2) the proportion between surface water and groundwater for irrigation is constant, and the irrigation from groundwater can be satisfied; (3) the external water use of Yuecheng Reservoir to other areas would be ignored in special years, and only the water distribution to irrigation areas of Minyou and Zhangnan in Handan City and Anyang City individually are considered; (4) since most of the water distribution of Yuecheng Reservoir is used to irrigate the three crops (wheat, corn, and cotton) of the two irrigation areas, other uses of the surface water in the irrigation areas would be ignored in this study. The objective of this study is to maximize the system benefit, which equals the net irrigation benefit minus the diversion cost and shortage penalty. Accordingly, the IIWRD model for this study system can be formulated as follows:

$$
\operatorname{Max} \lambda^{ \pm}
$$

subject to the following:

Constraint of system benefit:

$$
\begin{aligned}
& \sum_{i=1}^{15} \sum_{j=1}^{3} I T_{i j}^{ \pm} N B_{i j}^{ \pm}-\sum_{h=1}^{7} p_{h}\left(\frac{1}{2 H C^{ \pm} \sqrt{2 S C^{ \pm} D_{h}^{ \pm} / H C^{ \pm}+C^{ \pm} D_{h}^{ \pm}+\sqrt{S C^{ \pm} D_{h}^{ \pm} H C^{ \pm} / 2}}}\right) \\
& -\sum_{i=1}^{15} \sum_{j=1}^{3} \sum_{h=1}^{7} p_{h} I S_{i j h}^{ \pm} P C_{i j}^{ \pm} \geq f^{-}+\lambda^{ \pm}\left(f^{+}-f^{-}\right) .
\end{aligned}
$$

Constraint of water balance:

$$
+A Q_{h}^{+}-\lambda^{ \pm}\left(A Q_{h}^{+}-A Q_{h}^{-}\right), \quad \forall h .
$$

$$
\sum_{i=1}^{15} \sum_{j=1}^{3}\left(I T_{i j}^{ \pm}-I S_{i j h}^{ \pm}\right) Z Z X S_{i j}^{ \pm} \leq D_{h}^{ \pm}
$$

Constraint of minimum irrigation:

$$
I T_{i j}^{ \pm}-I S_{i j h}^{ \pm} \geq I T_{i j \min }^{-}+\lambda^{ \pm} \Delta I T_{i j \min }, \quad \forall i, j, h .
$$


Constraint of reservoir capacity:

$$
\begin{aligned}
& D_{h}^{ \pm}+A Q_{h}^{+}-\lambda^{ \pm}\left(A Q_{h}^{+}-A Q_{h}^{-}\right) \\
& -\sum_{i=1}^{15} \sum_{j=1}^{3}\left(I T_{i j}^{ \pm}-I S_{i j h}^{ \pm}\right) Z Z X S_{i j}^{ \pm} \leq R C^{ \pm}, \quad \forall h .
\end{aligned}
$$

Constraint of adjustable water diversion:

$$
D_{h}^{ \pm} \leq A D^{ \pm}, D_{h}^{ \pm} \geq 0, \quad \forall h
$$

Constraint of diversion batch:

$$
Q_{h}^{ \pm}=\sqrt{\frac{2 S C^{ \pm} D_{h}^{ \pm}}{H C^{ \pm}}}, \quad \forall h .
$$

Constraint of satisfaction degree:

$$
0 \leq \lambda^{ \pm} \leq 1
$$

The notation in models $(4 \mathrm{a})-(4 \mathrm{~h})$ is as follows:

$i$ : the irrigation subarea, $i=1,2, \ldots, 15$.

$j$ : the kind of crops, $j=1$ (wheat), 2 (corn), 3 (cotton).

$h$ : the inflow of Yuecheng Reservoir, $h=1,2, \ldots, 7$.

$p_{h}$ : the probability of inflow $h, p_{h}>0$ and $\sum_{h=1}^{h_{t}} p_{h}=1$.

$f^{ \pm}$: net benefit of the study system $\left(10^{6} \mathrm{RMB}\right)$.

$A D^{ \pm}$: adjustable water diversion from upstream reservoir $\left(10^{6} \mathrm{~m}^{3}\right)$.

$A Q_{h}^{ \pm}$: the water available for irrigation in Yuecheng Reservoir under inflow $h\left(10^{6} \mathrm{~m}^{3}\right)$.

$\stackrel{C}{ }^{ \pm}$: unit cost for water diversion $\left(\mathrm{RMB} / 10^{3} \mathrm{~m}^{3}\right)$.

$D_{h}^{ \pm}$: amount by which total water-allocation target is not met when the inflow is $h\left(10^{6} \mathrm{~m}^{3}\right)$.

$H C^{ \pm}$: holding cost of water per unit in the reservoir $\left(\mathrm{RMB} / 10^{3} \mathrm{~m}^{3}\right)$.

$I A_{i j h}^{ \pm}$: the actual irrigation quantity of crop $j$ in subarea $i$ when the inflow is $h$ (ha).

$I T_{i j}^{ \pm}$: the irrigation quota for crop $j$ in subarea $i$ (ha).

$I T_{i j \min }^{ \pm}$: the minimum planting quantity of crop $j$ in subarea $i$ (ha).

$I S_{i j h}^{ \pm}$: the unirrigation quantity of crop $j$ in subarea $i$ when the inflow is $h$ (ha).

$N B_{i j}^{ \pm}$: the net irrigation benefit for crop $j$ in subarea $i$ per unit of surface water allocated $\left(10^{3} \mathrm{RMB} / \mathrm{ha}\right)$.

$Q_{h}^{ \pm}$: the diversion batch under the inflow $h\left(\mathrm{~m}^{3}\right)$.

$P C_{i j}^{ \pm}$: the reduction of net benefit for crop $j$ in subarea $i$ when per unit of surface water not delivered $\left(10^{3} \mathrm{RMB} /\right.$ ha).
$R C^{ \pm}$: storage capacity of the reservoir $\left(10^{6} \mathrm{~m}^{3}\right)$.

$S C^{ \pm}$: setup cost for water diversion one batch (RMB). $T_{h}^{ \pm}$: the diversion period under the inflow $h$ (hour).

$Z Z X S_{i j}^{ \pm}$: the water demand of crop $j$ per unit in subarea $i\left(10^{6} \mathrm{~m}^{3} / \mathrm{ha}\right)$.

In models (4a)-(4h), $D_{h}^{ \pm}$and $I S_{i j h}^{ \pm}$are decision variables, which are influenced by the inflow of Yuecheng Reservoir. The detailed solution process for solving models (4a)-(4h) can be summarized as follows:

Step 1. Transform models $(4 \mathrm{a})-(4 \mathrm{~h})$ into two submodels, where submodel $(A)$ with $\lambda^{+}$corresponding to $f^{+}$should be formulated first.

Step 2. Solve submodel $(A)$ and obtain the solutions of $\lambda_{\text {opt }}^{+}, D_{h \text { opt }}^{+}, Q_{h \text { opt }}^{+}$, and $I S_{i j h}^{-}$.

Step 3. Calculate $I A_{i j h o p t}^{+}$according to $I A_{i j h}^{+}=I T_{i j h}^{+}-$ $I S_{i j h}^{-}$and obtain $T_{h o p t}^{+}$and $f_{\text {opt }}^{+}$by $f_{\text {opt }}^{+}=\sum_{j=1}^{k_{1}} c_{j}^{+} x_{j}^{+}+$ $\sum_{j=k_{1}+1}^{n_{1}} c_{j}^{+} x_{j}^{-}-\sum_{h=1}^{v}\left(1 / 2 H C^{-} \sqrt{2 S C^{-} Y_{h}^{-} / H C^{-}}+C^{-} Y_{h}^{-}+\right.$ $\left.\sqrt{S C^{-} Y_{h}^{-} H C^{-} / 2}\right)$

Step 4. Formulate submodel $(B)$ with $\lambda^{-}$corresponding to $f^{-}$.

Step 5. Solve submodel $(B)$ and obtain the solutions of $\lambda_{\text {opt }}^{-}, D_{h \text { opt }}^{-}, Q_{h \text { opt }}^{-}$, and $I S_{i j h}^{+}$.

Step 6. Calculate $I A_{i j h \text { opt }}^{-}$according to $I A_{i j h}^{-}=I T_{i j h}^{-}-I S_{i j h}^{+}$and obtain $T_{h \text { opt }}^{-}$and $f_{\text {opt }}^{-}$by $f_{\mathrm{opt}}^{-}=\sum_{j=1}^{k_{1}} c_{j}^{-} \quad x_{j}^{-}+\sum_{j=k_{1}+1}^{n_{1}} c_{j}^{-} x_{j}^{+}-\sum_{h=1}^{v} p_{h}\left(1 / 2 H C^{+}\right.$ $\left.\sqrt{2 S C^{+} Y_{h}^{+} / H C^{+}}\right)+C^{+} Y_{h}^{+}+\sqrt{S C^{+} Y_{h}^{+} H C^{+} / 2}$.

Step 7. Integrate the two submodel solutions to obtain the optimal solutions for the IFTSIP model, which can be expressed as $D_{\text {topt }}^{ \pm}=\left[D_{\text {topt }}^{-}, D_{\text {topt }}^{+}\right], \quad Q_{t o p t}^{ \pm}=$ $\left[Q_{\text {topt }}^{-}, Q_{\text {topt }}^{+}\right], \quad T_{h \text { opt }}^{ \pm}=\left[T_{\text {hopt }}^{ \pm}, T_{\text {hopt }}^{ \pm}\right], \quad I S_{i j h o p t}^{ \pm}=$ $\left[I S_{i j h \mathrm{opt}}^{-}, I S_{i j h \mathrm{opt}}^{+}\right], \quad I A_{i j h \mathrm{opt}}^{ \pm}=\left[I A_{i j h \mathrm{opt}}^{-}, I A_{i j h \mathrm{opt}}^{+}\right]$, $\lambda_{\text {opt }}^{ \pm}=\left[\lambda_{\text {opt }}^{-}, \lambda_{\text {opt }}^{+}\right]$, and $f_{\text {opt }}^{ \pm}=\left[f_{\text {opt }}^{-}, f_{\text {opt }}^{+}\right]$.

Step 8. Stop.

\section{Data Acquisition and Analysis}

In this study, the surface water irrigation target of three kinds of crops in each subarea is shown in Table 1 [41]. Table 2 presents the net irrigation benefit and penalty of each crop in every subarea, which are expressed as different intervals based on the various times and spaces. For example, the net irrigation benefit of corn in $\mathrm{Ci}$ County is $[2.78,3.30] \times 10^{3} \mathrm{RMB} / \mathrm{ha}$, which is obtained by the following calculation: (a) the unit yield of corn in Ci County is from $6.10 \mathrm{t} / \mathrm{ha}$ to $6.25 \mathrm{t} / \mathrm{ha}$, in which $6.10 \mathrm{t} / \mathrm{ha}$ and $6.25 \mathrm{t} / \mathrm{ha}$ present the minimum and maximum values of the unit yield, respectively; (b) the net benefit of corn is from $570 \mathrm{RMB} / \mathrm{t}$ to $660 \mathrm{RMB} / \mathrm{t}$; and (c) the ratio of corn by irrigation is about 0.8 . Therefore, the minimum value of net irrigation benefit of unit yield of corn in $\mathrm{Ci}$ County is $2.78 \mathrm{RMB} / \mathrm{ha}$ $[6.10 \mathrm{t} / \mathrm{ha} \times 0.8 \times 570 \mathrm{RMB} / \mathrm{t}]$, which would be the lower bound of the interval, while the maximum value is $3.30 \mathrm{RMB} / \mathrm{ha} \quad[6.25 \mathrm{t} / \mathrm{ha} \times 0.8 \times 660 \mathrm{RMB} / \mathrm{t}]$, which 
TABLe 1: Surface water irrigation targets.

\begin{tabular}{lccc}
\hline \multirow{2}{*}{ Subarea } & \multicolumn{3}{c}{ Irrigation target, $I T_{i j}^{ \pm}($ha) } \\
& Wheat & Corn & Cotton \\
\hline QZ & {$[1730,1830]$} & {$[1770,1870]$} & {$[870,1000]$} \\
FX & {$[2570,2680]$} & {$[2000,2200]$} & {$[1050,1150]$} \\
GP & {$[1430,1570]$} & {$[1050,1200]$} & {$[550,650]$} \\
CA & {$[2730,2925]$} & {$[1716,1820]$} & {$[1820,2080]$} \\
WX & {$[2550,2670]$} & {$[2280,2490]$} & {$[144,174]$} \\
CX & {$[1145,1207.5]$} & {$[1145,1250]$} & {$[95,117.5]$} \\
LZ & {$[4860,5140]$} & {$[4400,4740]$} & {$[600,734]$} \\
DM & {$[2070,2175]$} & {$[990,1072.5]$} & {$[55.5,64.5]$} \\
WF & {$[1980,2025]$} & {$[2010,2160]$} & {$[130,150]$} \\
BG & {$[420,450]$} & {$[420,450]$} & {$[7,10]$} \\
YD & {$[645,675]$} & {$[630,675]$} & {$[4.5,6.5]$} \\
LA & {$[1950,2070]$} & {$[1590,1680]$} & {$[225,255]$} \\
AY & {$[8000,8667.5]$} & {$[9167.5,10000]$} & {$[450,492.5]$} \\
NH & {$[1354.7,1430.6]$} & {$[437,483]$} & {$[89.7,128.8]$} \\
KFQ & {$[255,270]$} & {$[240,270]$} & 0 \\
\hline
\end{tabular}

TABLE 2: Net irrigation benefits and penalties.

\begin{tabular}{lccr}
\hline & Wheat & Corn & \multicolumn{1}{c}{ Cotton } \\
\hline Net irrigation benefit when water & demand is met, $N B_{i j}^{ \pm}$ & $\left(10^{3} \mathrm{RMB} / \mathrm{ha}\right)$ \\
QZ & {$[2.18,2.67]$} & {$[2.75,3.25]$} & {$[1.91,3.43]$} \\
FX & {$[2.38,2.90]$} & {$[3.44,4.09]$} & {$[2.54,4.41]$} \\
GP & {$[2.34,2.85]$} & {$[3.35,3.96]$} & {$[1.91,3.31]$} \\
CA & {$[2.54,3.14]$} & {$[3.50,4.17]$} & {$[2.01,3.43]$} \\
WX & {$[2.24,2.74]$} & {$[2.73,3.25]$} & {$[1.84,3.19]$} \\
CX & {$[2.20,2.69]$} & {$[2.78,3.30]$} & {$[1.88,3.38]$} \\
LZ & {$[2.55,3.11]$} & {$[3.53,4.20]$} & {$[1.98,3.43]$} \\
DM & {$[2.32,2.83]$} & {$[3.00,3.54]$} & {$[1.40,2.57]$} \\
WF & {$[2.34,2.90]$} & {$[2.73,3.22]$} & {$[0.96,1.96]$} \\
BG & {$[2.44,3.04]$} & {$[3.10,3.75]$} & {$[1.98,3.68]$} \\
YD & {$[2.34,2.90]$} & {$[3.25,3.91]$} & {$[2.35,4.41]$} \\
LA & {$[1.33,1.73]$} & {$[2.28,2.80]$} & {$[1.18,2.45]$} \\
AY & {$[2.42,3.00]$} & {$[2.91,3.46]$} & {$[1.10,2.33]$} \\
NH & {$[2.28,2.81]$} & {$[3.05,3.62]$} & {$[1.91,3.68]$} \\
KFQ & {$[2.55,3.18]$} & {$[3.00,3.59]$} & \\
\hline & Penalty & when water is not $]$ & \\
QZ & {$[3.27,3.42]$} & {$[3.65,3.75]$} & {$[3.83,3.93]$} \\
FX & {$[3.50,3.65]$} & {$[4.49,4.59]$} & {$[4.81,4.91]$} \\
GP & {$[3.45,3.60]$} & {$[4.36,4.46]$} & {$[3.71,3.81]$} \\
CA & {$[3.74,3.89]$} & {$[4.57,4.67]$} & {$[3.83,3.93]$} \\
WX & {$[3.34,3.49]$} & {$[3.65,3.75]$} & {$[3.59,3.69]$} \\
CX & {$[3.29,3.44]$} & {$[3.70,3.80]$} & {$[3.78,3.88]$} \\
LZ & {$[3.71,3.86]$} & {$[4.60,4.70]$} & {$[3.83,3.93]$} \\
DM & {$[3.43,3.58]$} & {$[3.94,4.04]$} & {$[2.97,3.07]$} \\
WF & {$[3.50,3.65]$} & {$[3.62,3.72]$} & {$[2.36,2.46]$} \\
BG & {$[3.64,3.79]$} & {$[4.15,4.25]$} & {$[4.08,4.18]$} \\
YD & {$[3.50,3.65]$} & {$[4.31,4.41]$} & {$[4.81,4.91]$} \\
LA & {$[2.33,2.48]$} & {$[3.20,3.30]$} & {$[2.85,2.95]$} \\
AY & {$[3.60,3.75]$} & {$[3.86,3.96]$} & {$[2.73,2.83]$} \\
NH & {$[3.41,3.56]$} & {$[4.02,4.12]$} & {$[4.08,4.18]$} \\
KFQ & {$[3.78,3.93]$} & {$[3.99,4.09]$} & 0 \\
\hline & & & \\
\hline
\end{tabular}

would be the upper bound of the interval. The net irrigation benefits of other crops in each subarea also can be obtained by the same method. The amount of adjustable water diversion from upstream reservoirs is $[112+23,112+37] \times 106 \mathrm{~m}^{3}$, which is estimated based on the sum of annual abandoned water from Zhangze Reservoir and Guanghe and Back bay Reservoir from 1960 to 2004
[38]. Since the irrigation areas of Minyou and Zhangnan are the important grain production base in Handan City and Anyang City individually, the minimum planting quantity of each crop in every subarea is the lowest irrigation area in order to ensure the local ecology and grain yield, whose value accounts for $40 \%-50 \%$ percent of its total planting area [42]. The setup cost for water diversion in this paper mainly considers the travelling expenses for diversion agreement per time, whose value is $[260,370] \mathrm{RMB}$ by calculating the costs of tickets, meals, and accommodation. According to "Notice on the trial implementation of financial benchmark yield and annual operating standard rates" [43], the holding cost of Yuecheng Reservoir is $[30,40] \mathrm{RMB} / 10^{3} \mathrm{~m}^{3}$ by calculating the ratio between the sum of three costs: maintenance fee $\left(41.5 \times 10^{6} \mathrm{RMB}\right)$, salary and welfare funds for managers $\left(12.13 \times 10^{9} \mathrm{RMB}\right)$, the reservoir management fee $\left(1.82 \times 10^{6} \mathrm{RMB}\right)$, and the annual runoff of the reservoir $[44,45]$. In addition, due to the lack of related information, the distribution of water supply of Yuecheng Reservoir for irrigation is difficult to obtain. Based on data statistics, the range of $[0,302.71106] \mathrm{m}^{3}$ is selected as the available water for irrigation, and the water supply level for irrigation can be divided into seven discrete intervals, which are very low (VL, $17 \%$ ), low (L, 9\%), low to medium (L-M, 14\%), medium (M, $25 \%)$, medium to high $(\mathrm{M}-\mathrm{H}, 20 \%)$, high $(\mathrm{H}, 10 \%)$, and very high ( $\mathrm{VH}, 5 \%$ ), and the corresponding water quantities are 0 , $[29.58,46.39] \times 10^{6} \mathrm{~m}^{3}, \quad[83.39,108.05] \times 10^{6} \mathrm{~m}^{3}$, $[108.05,127.25] \times 10^{6} \mathrm{~m}^{3}, \quad[127.25,148.16] \times 10^{6} \mathrm{~m}^{3}$, $[198.26,222.76] \times 10^{6} \mathrm{~m}^{3}$, and $[233.06,302.71] \times 10^{6} \mathrm{~m}^{3}$ [39].

\section{Result Analysis and Discussion}

6.1. Water Diversion Analysis. When the random event of water supply happened and makes the local water demand not to meet, the corrective action of water diversion of Yuecheng Reservoir from upstream would be taken to reduce the water shortage. The total diversion quantity is related to various inflows, irrigation quota (expected irrigation objectives), and the minimum planting quantity. If the manager is optimistic about the inflow level (upper bound), adjustable diversion (upper bound), and diversion cost (lower bound) and promises to farmers with the upper bound of the irrigation quota, the water diversion from upstream reservoirs will be more; accordingly, the crop areas of water shortage will be less, and vice versa. The diversion batch is mainly affected by the total amount of water diversion, setup cost, and holding cost, meaning the quantity per time. The diversion period is the ratio of diversion batch and the total amount of water diversion, indicating the time interval between two water diversions.

Table 3 shows the optimal scheme of water diversion of Yuecheng Reservoir from upstream reservoir under different inflows, including total diversion quantity, diversion batch, and period. It can be seen that the total amount of water diversion in the reservoir gradually decreases with the increase in inflow level, which results in the same tendency to diversion batch. This indicates that when the reservoir inflow is high, the agricultural water demand in each subarea can be 
TABLE 3: Results of water diversion.

\begin{tabular}{lccr}
\hline Inflow levels & Diversion quantity $\left(10^{6} \mathrm{~m}^{3}\right)$ & Diversion batch $\left(10^{3} \mathrm{~m}^{3}\right)$ & Diversion period $(\mathrm{h})$ \\
\hline VL & {$[135.00,149.00]$} & {$[49.30,51.79]$} & {$[3.04,3.20]$} \\
L & {$[135.00,149.00]$} & {$[49.30,51.79]$} & {$[3.04,3.20]$} \\
L-M & {$[92.31,109.48]$} & {$[40.76,44.39]$} & {$[3.55,3.87]$} \\
M & {$[72.94,84.97]$} & {$[36.23,39.11]$} & {$[4.03,4.35]$} \\
M-H & {$[52.08,65.72]$} & {$[30.62,34.39]$} & {$[4.58,5.15]$} \\
H & - & - & - \\
VH & - & - & - \\
\hline
\end{tabular}

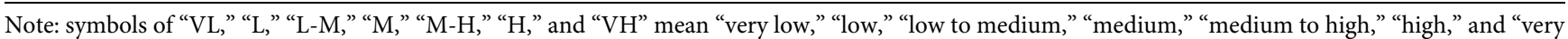
high," respectively.

satisfied without water diversion. Conversely, when the inflow level is low, the reservoir needs a lot of water diversion to ensure the minimum irrigation. For example, when the inflow level is very low and low, the total amount of water diversion is $[135,149] \times 10^{6} \mathrm{~m}^{3}$ and $[135,149] \times$ $10^{6} \mathrm{~m}^{3}$, individually, in which $135 \times 10^{6} \mathrm{~m}^{3}$ and $149 \times 10^{6} \mathrm{~m}^{3}$ are the lower and upper bounds of adjustable water diversion, respectively, while the related diversion batch is [49.30, $51.79] \times 10^{3} \mathrm{~m}^{3}$. Under this condition, although the diversion batch is oppositely larger, the diversion period is relatively short because the total water diversion is the largest of all inflow levels. This indicates that the continued water shortage in the study area can be solved only by many times of water diversion. Similar characteristics can be found under other inflow levels. Totally, it can be seen that when the total water diversion decreases gradually, the diversion period will become relatively long. The symbol of "/" in Table 3 indicates the diversion period does not exist at levels of high and very high.

6.2. Water-Allocation Analysis. After taking water diversion, the optimized water allocations to irrigated crops under different inflow levels also can be obtained by the proposed IIWRD model. It is noted that the optimized irrigation quantity is influenced by not only the different inflow levels and water diversion quantity but also the net irrigation benefits and water shortage penalties of crops. Figure 2 shows the optimal irrigation quantity of wheat in each subarea under various inflow levels. It can be seen that when the inflow level is very low, there is still a big difference between the total water diversion and expected water quota even though the total amount of water diversion takes the maximum value within the allowable range of adjustable water diversion. Therefore, the actual irrigation quantity of wheat in most of subareas only can get the minimum irrigation quantity, which is $[692,915]$ ha, $[1028,1340]$ ha, $[572,785]$ ha, $\quad[1020,1335]$ ha, $\quad[458,603.75]$ ha, $[828,1087.5]$ ha, $[792,1012.5],[168,225]$ ha, $[258,337.5]$ ha, [780, 1035]ha, [3200, 4333.75]ha, and [541.6, 715.3] ha in subareas of Quzhou, Feixiang, Guangping, Weixian, Cixian, Daming, Wenfeng, Beiguan, Yindu, Long'an, Anyang, and Neihuang, respectively. When the inflow level is low to medium, medium, and medium to high, the actual irrigation quantity of wheat in all subareas can reach the upper bound of expected water quota, which is $1830 \mathrm{ha}, 2680 \mathrm{ha}, 1570 \mathrm{ha}$, 2925 ha, 2670 ha, 1207.5 ha, 5140 ha, 2175 ha, 2025 ha,
450 ha, 675 ha, 2070 ha, 8667.5 ha, 1430.5 ha, and 270 ha in subareas of Quzhou, Feixiang, Guangping, Cheng'an, Weixian, Cixian, Linzhang, Daming, Wenfeng, Beiguan, Yindu, Long'an, Anyang, Neihuang, and Kaifaqu, respectively. Meanwhile, the lower bound of expected water quota for wheat can be acquired in more and more subareas with the total increasing water supply. When the inflow level is high and very, both of the upper and lower bounds of expected water quota for wheat in all subareas can be reached simultaneously.

Figure 3 illustrates the optimal irrigation quantity of corn in each subarea under various inflow levels. For the crop of corn, the actual irrigation quantity has acquired both upper and lower bounds of the expected water quota in all subareas when the inflow level is from very low to very high. Their specific values are $[1770,1870]$ ha, [2000, 2200]ha, $[1050,1200]$ ha, $\quad[1716,1820]$ ha, $\quad[2280,2490]$ ha, $[1145,1250]$ ha, $\quad[4400,4740]$ ha, $\quad[990,1072.5]$ ha, $[2010,2160]$ ha, $[420,450]$ ha, $[630,675]$ ha, $[1590,1680]$ ha, [9167.5, 10000]ha, [437, 483]ha, and [240,270]ha in subareas of Quzhou, Feixiang, Guangping, Cheng'an, Weixian, Cixian, Linzhang, Daming, Wenfeng, Beiguan, Yindu, Long'an, Anyang, Neihuang, and Kaifaqu, individually. Because of its higher net irrigation benefit and higher water shortage penalty, corn has priority to be irrigated over wheat and cotton in the same subarea under every inflow level. Therefore, with the addition of water diversion, the upper and lower bounds of the expected water quota for corn would be firstly reached even when the inflow level is very low.

Figure 4 depicts the optimal irrigation quantity of cotton in each subarea under various inflow levels. After comparing, although cotton and wheat have similar average net irrigation benefits, the water shortage penalty of cotton is higher than that of wheat in subareas of Quzhou, Feixiang, Guangping, Cheng'an, Weixian, Cixian, Linzhang, Beiguan, Yindu, and Neihuang. Accordingly, in these subareas, the expected quota for cotton can be met prior to that of wheat when the inflow level is from very low to very high, which are $[870,1000]$ ha, $[1050,1150]$ ha, $[550,650]$ ha, $[1820,2080]$ ha, $[144,174]$ ha, $[95,117.5]$ ha, $[600,734]$ ha, $[7,10]$ ha, $[4.5,6.5]$ ha, $[225,255] \mathrm{ha}$, and $[89.7,128.8]$ ha, respectively. For the cotton in subareas of Daming, Wenfeng, Long'an, and Anyang, due to their lower net irrigation benefits and lower water shortage penalties than that of the other subareas, their expected quota would be considered later when 


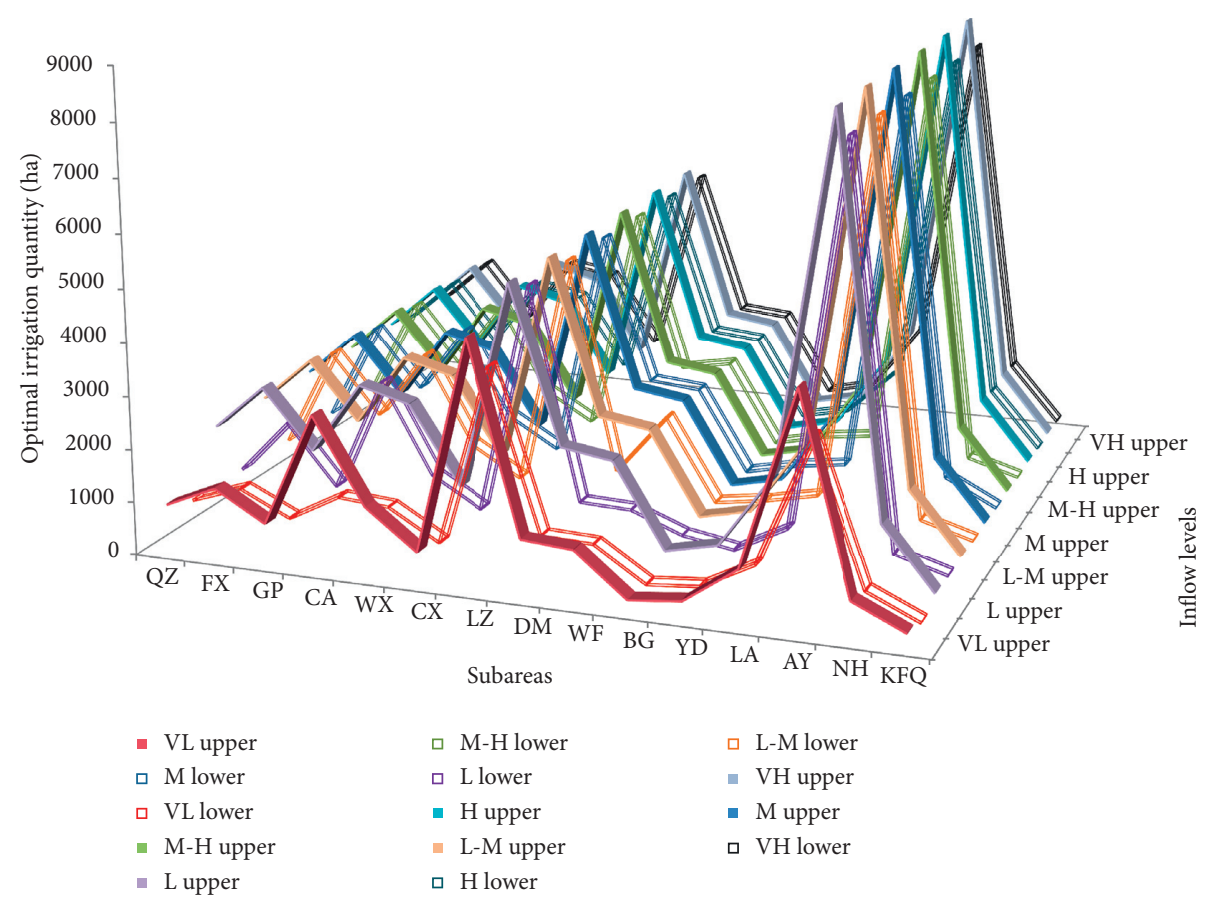

FIGURE 2: Optimal irrigation quantity of wheat in each subarea under different inflow levels.

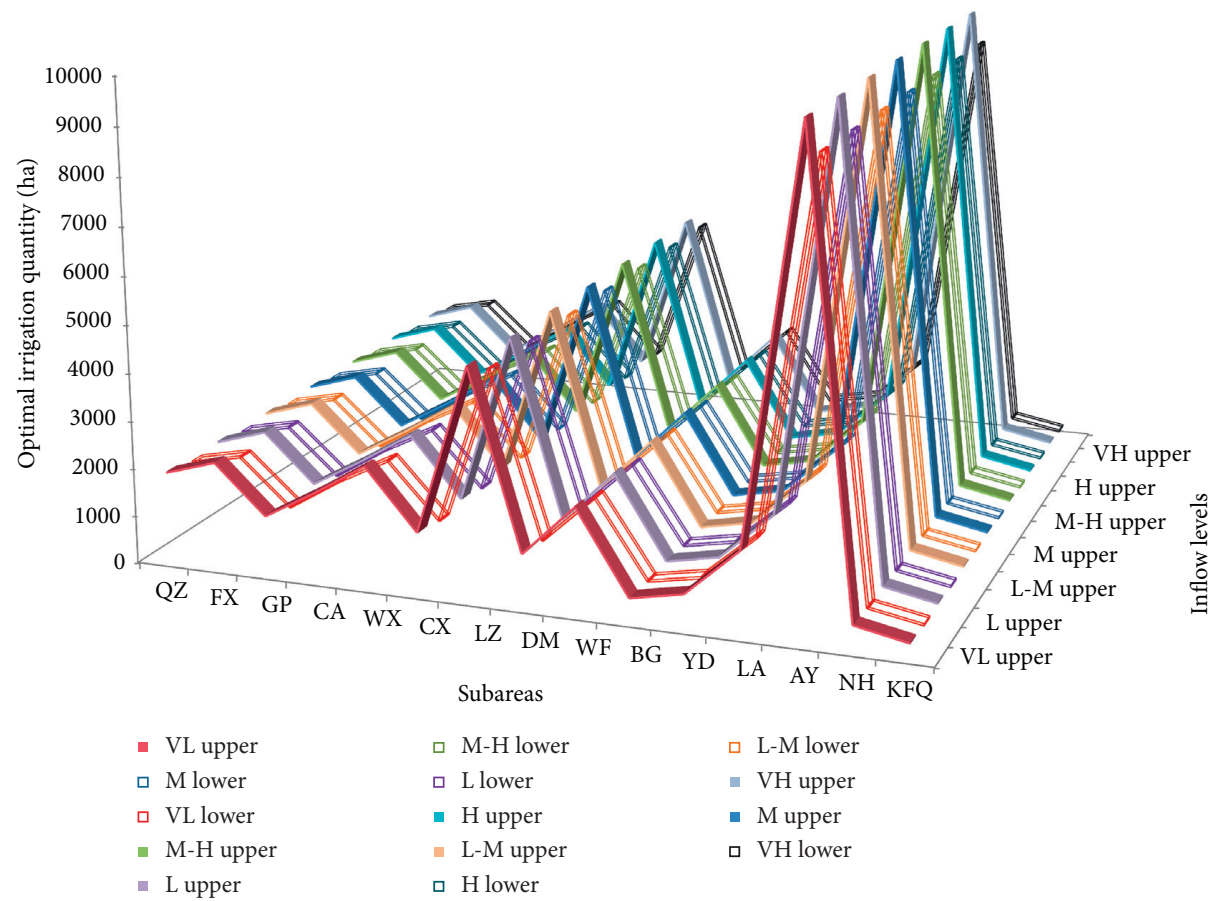

Figure 3: Optimal irrigation quantity of corn in each subarea under different inflow levels.

the inflow level is very low, whose optimal irrigation quantities are $[22.2,64.5]$ ha, $[52,75]$ ha, $[90,255]$ ha, and $[180,492.5]$ ha individually; When the inflow level is from low to medium to high, their optimal irrigation quantities are $[22.2,64.5]$ ha, $[52,150]$ ha, $[90,255]$ ha, and [180,492.5] ha individually; until when the inflow level is high and very high, both the upper and lower bounds of their expected quotas can be met simultaneously, which are
$[55.5,64.5]$ ha, $\quad[130,150]$ ha, $\quad[225,255]$ ha, $\quad$ and $[450,492.5]$ ha, respectively.

6.3. System Benefit and Uncertainty Analysis. The expected maximum benefit of this study is $[165.12,231.3] \times 10^{6} \mathrm{RMB}$ by solving the IIWRD model, while the corresponding satisfaction degree $\left(\lambda_{\mathrm{opt}}^{ \pm}\right)$is $[0.032,0.972]$. This interval result 


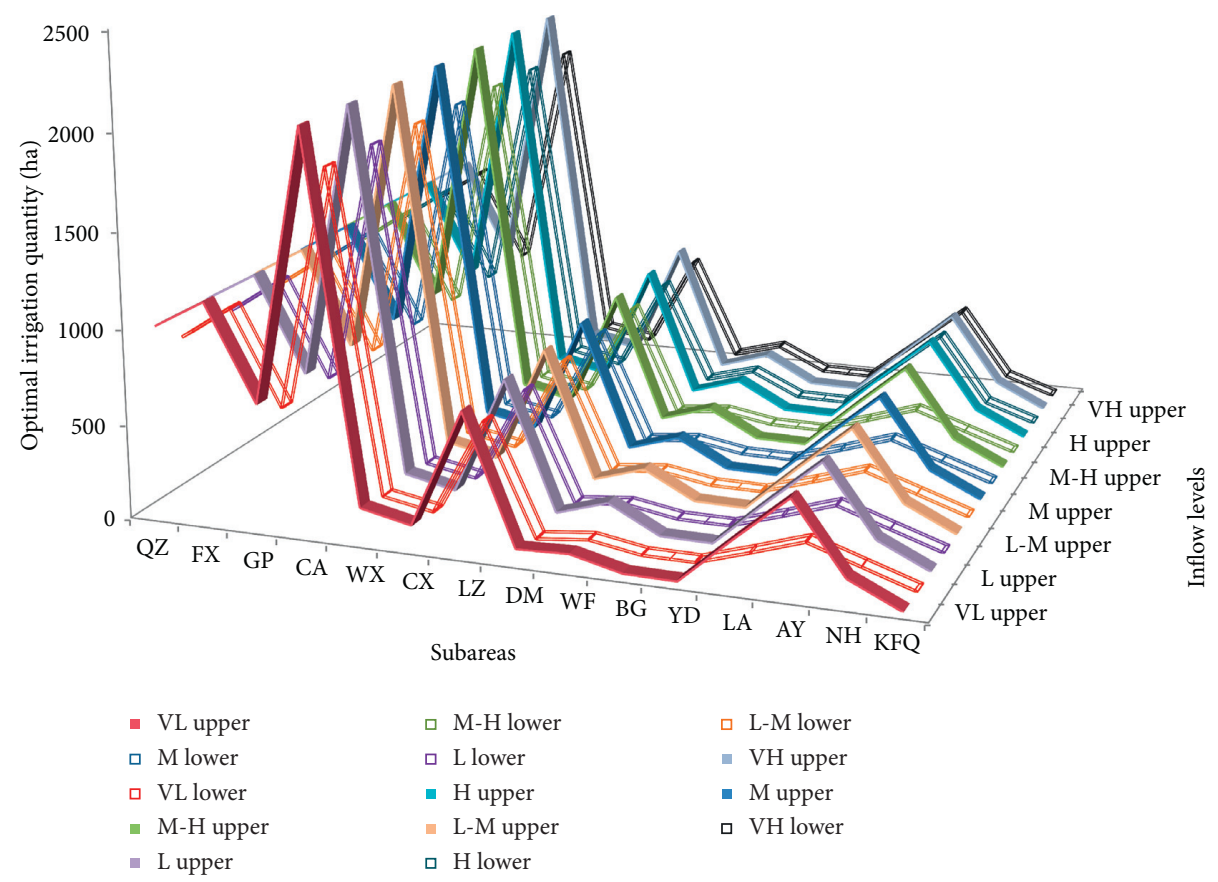

FIGURE 4: Optimal irrigation quantity of cotton in each subarea under different inflow levels.

indicates that when the actual value of each variable fluctuates in its interval, the system benefit would correspondingly change between $f_{\mathrm{opt}}^{+}$and $f_{\mathrm{opt}}^{-}$under different satisfaction degrees. When the inflow level, potential probability distribution, and benefit and punishment and other economic data are given different values, the related decision would change accordingly between the upper and lower bounds of the solution interval. $\lambda^{ \pm}$means the satisfaction degree of decision-makers in balancing the environment and economic benefit. In detail, $\lambda_{\text {opt }}^{-}=0.032$ is consistent with the lower system benefit $\left(f_{\mathrm{opt}}^{-}=165.12 \times 10^{6} \mathrm{RMB}\right)$, indicating the maximum satisfaction degree under inferior situation. In contrast, $\lambda_{\text {opt }}^{+}=0.972$ is consistent with the higher system benefit $\left(f_{\mathrm{opt}}^{+}=231.3 \times 10^{6} \mathrm{RMB}\right)$, indicating the maximum satisfaction degree of the superior situation. Therefore, the solution of $\lambda_{\text {opt }}^{ \pm}$represents the degree of meeting the system objectives and constraints under uncertainty.

In addition, the uncertain unit diversion cost expressed as FIN (fuzzy-interval number) directly influences the system benefit and its related satisfaction degree. To clearly understand their relationship, the results under various unit diversion costs are obtained by solving the IIWRD model (as shown in Table 4). It is noted that "S1," "S2," "S3," "S4," and "S5" indicate different scenarios in consistent with five-unit diversion cost, respectively. In detail, the scenario of "S1" represents the lower and upper bounds of the unit diversion cost are fuzzy $\left(\widetilde{C}^{ \pm}=\left[{\underset{\sim}{C}}^{-},{\underset{\sim}{+}}^{+}\right]\right)$, and the corresponding system benefit and satisfaction degree of this fuzzy decision are $f_{\mathrm{opt}}^{ \pm}=[165.12,231.30] \times 10^{6} \mathrm{RMB}$ and $\lambda_{\mathrm{opt}}^{ \pm}=[0.032$, $0.972]$ individually. The scenario of "S2" indicates the lower and upper bounds of the unit diversion cost are determinate $\left(\widetilde{C}^{ \pm}=\left[\underline{C}^{-}, \bar{C}^{+}\right]\right.$, meaning the fuzzy boundary is simplified as certain information); accordingly, the system benefit and satisfaction degree are $f_{\mathrm{opt}}^{ \pm}=[164.85,231.63] \times 10^{6} \mathrm{RMB}$ and $\lambda_{\text {opt }}^{ \pm}=[0.028,0.977]$, respectively. When the lower bound of unit diversion cost is determinate but the upper bound is fuzzy (the scenario of "S3," $\underline{C}^{ \pm}=\left[\bar{C}^{-}, \underline{\sim}^{+}\right]$), the system benefit and satisfaction degree are $f_{\text {opt }}^{ \pm}=[165.12,233.23] \times 10^{6} \mathrm{RMB}$ and $\lambda_{\mathrm{opt}}^{ \pm}=[0.032,0.999]$, individually. When the unit diversion cost has fuzzy lower bound and certain upper bound (the scenario of "S4," $\left.\underline{C}^{ \pm}=\left[\underline{C}^{-}, \underline{C}^{+}\right]\right)$, the system benefit and satisfaction degree are $f_{\mathrm{opt}}^{ \pm}=[165.15,231.30] \times 10^{6} \mathrm{RMB}$ and $\lambda_{\mathrm{opt}}^{ \pm}=[0.032$, $0.972]$, respectively. When the upper bound of unit diversion cost is fuzzy but the lower bound is certain and close to the lower boundary of lower interval (the scenario of "S5," $\left.\underline{C}^{ \pm}=\left[\underline{C}^{-}, \underline{C}^{+}\right]\right)$, the system benefit and satisfaction degree are $f_{\mathrm{opt}}^{ \pm}=[165.42,231.63] \times 10^{6} \mathrm{RMB}$ and $\lambda_{\mathrm{opt}}^{ \pm}=[0.036$, $0.977]$, individually. In scenarios of "S3," "S4," and "S5," both of the lower bound of system benefit and the satisfaction degree are higher than or equal to the result of "S1" scenario $\left(f_{\text {opt }}^{-}=165.15 \times 10^{6} \mathrm{RMB}\right.$ and $\left.\lambda_{\text {opt }}^{-}=0.032\right)$. This is because of the lower bound of uncertain unit diversion cost and/or the relaxation of allowable default in the inferior situation. The relaxation of system constraints represents an increase in the risk of constraints default. Under the lower risk of constraints default, the decision can be obtained with lower system benefit but higher system credibility. Higher system benefit will result in a larger risk of system constraints default.

Table 5 shows the total amount of water diversion under scenarios of different inflow levels and various unit diversion costs. It can be known that when the inflow level is very low and low, due to the serious water shortage, although the unit diversion cost changes ( $\mathrm{S} 1$ : $[27.89,32.38] \mathrm{RMB} / 10^{3} \mathrm{~m}^{3}, \mathrm{~S} 2$ : 
TABLE 4: System benefit and satisfaction degree under different scenarios of unit diversion cost.

\begin{tabular}{lccccc}
\hline & & \multicolumn{3}{c}{ Scenarios of unit diversion cost } \\
& S1 & S2 & S3 & S4 & S5 \\
\hline System benefit $\left(10^{6} \mathrm{RMB}\right)$ & {$[165.12,231.30]$} & {$[164.85,231.63]$} & {$[165.12,233.23]$} & {$[165.15,231.30]$} & {$[165.42,231.63]$} \\
Satisfaction degree & {$[0.032,0.972]$} & {$[0.028,0.977]$} & {$[0.032,0.999]$} & {$[0.032,0.972]$} & {$[0.036,0.977]$} \\
\hline
\end{tabular}

TABle 5: Diversion quantity under different scenarios of inflow levels and unit diversion cost $\left(10^{6} \mathrm{~m}^{3}\right)$.

\begin{tabular}{lccccc}
\hline Inflow levels & \multicolumn{3}{c}{ Scenarios of unit diversion cost } \\
& S1 & S2 & S3 & S4 & S5 \\
\hline VL & {$[135.00,149.00]$} & {$[135.00,149.00]$} & {$[135.00,149.00]$} & {$[135.00,149.00]$} & {$[135.00,149.00]$} \\
L & {$[135.00,149.00]$} & {$[135.00,149.00]$} & {$[135.00,149.00]$} & {$[135.00,149.00]$} & {$[135.00,149.00]$} \\
L-M & {$[92.31,109.48]$} & {$[92.22,109.60]$} & {$[92.31,110.16]$} & {$[92.32,109.48]$} & {$[92.42,109.60]$} \\
M & {$[72.94,84.97]$} & {$[72.87,85.06]$} & {$[72.87,85.50]$} & {$[72.95,84.97]$} & {$[73.02,85.06]$} \\
M-H & {$[52.08,65.72]$} & {$[52.00,65.82]$} & {$[52.08,66.30]$} & {$[52.09,65.72]$} & {$[52.17,65.82]$} \\
H & 0 & 0 & 0 & 0 & 0 \\
VH & 0 & 0 & 0 & 0 & 0 \\
\hline
\end{tabular}

$[24,36] \mathrm{RMB} / 10^{3} \mathrm{~m}^{3}, \mathrm{~S} 3:[28,32.38] \mathrm{RMB} / 10^{3} \mathrm{~m}^{3}, \mathrm{~S} 4:[27.89$, 32] $\mathrm{RMB} / 10^{3} \mathrm{~m}^{3}$, and $\mathrm{S} 5:[24,28.43] \mathrm{RMB} / 10^{3} \mathrm{~m}^{3}$ ), the total amount of water diversion has not changed, and all are $[135,149] \times 10^{6} \mathrm{~m}^{3}$. This indicates that, in the case of severe water shortage, the influence of unit diversion cost can be neglected in order to ensure the minimum irrigation quantity of each crop in every subarea. When the inflow level is lowmedium, medium, and medium-high, the total amount of water diversion is fluctuated as the unit diversion cost changes. For example, when the inflow level is low-medium, the total water diversion of five scenarios is $[72.94,84.97] \times$ $10^{6} \mathrm{~m}^{3}, \quad[72.872,85.06] \times 10^{6} \mathrm{~m}^{3}, \quad[72.94,85.50] \times 10^{6} \mathrm{~m}^{3}$, $[72.95,84.97] \times 10^{6} \mathrm{~m}^{3}$, and $[73.02,85.06] \times 10^{6} \mathrm{~m}^{3}$, respectively; similar characteristics exist in the solutions of total water diversion under the inflow level of medium and medium-high. When the inflow level is high and very high, the total water diversion is zero. It can be seen that when the inflow level is extreme, the small fluctuation of the unit diversion cost has no effect on the total water diversion. When the inflow level is normal in scenarios of "S3"-"S5," the lower bound of total water diversion increases with the decrease in the unit diversion cost, corresponding to the gradual increase in the satisfaction degree; the upper bound of total water diversion firstly decreases and then increases, in accordance with the satisfaction degree change. According to the results, it can be obtained that the influence of unit diversion cost changes on the system benefit and total water diversion needs to be considered when the inflow level is normal situation.

\section{Conclusions}

In this study, an inexact inventory theory-based water resources distribution (IIWRD) model has been proposed for the system of water distribution of a reservoir. This method is developed based on three techniques of ITSP, IFMP, and inventory theory. Therefore, the IIWRD model can not only indicate various uncertainties expressed as discrete intervals, probabilistic distributions, membership functions, and their combinations but also provide recourse measures for the expected objectives against different random events happened and the corresponding specific water diversion, including total diversion quantity, diversion batch size, and period. Meanwhile, it also can offer the manager the optimal schemes under different scenarios in realizing the maximum system benefit and help decision-makers to avoid the risk of water shortage. In addition, this method can also provide the system benefit under different satisfaction degrees and related schemes of water diversion and agricultural water allocation and a narrow solution range, which is convenient for decision-makers to make decisions.

A case of Yuecheng Reservoir in the Zhanghe River Basin diverting water from upstream and allocating water to agriculture in Handan City and Anyang City has been studied for demonstrating applicability of the proposed methodology. Through using the inventory theory, not only the optimal schemes of water diversion (the total diversion quantity, diversion batch size, and period) under different inflow levels are obtained but also the actual irrigation quantity of each crop is gained. They will help generate desired policies for managers with maximized system benefit and satisfaction degree. Moreover, a variety of uncertainties in the system is well addressed by the advanced IIWRD model. Therefore, the obtained results can effectively help the reservoir managers to solve the problems of water diversion and allocation and set up the optimal management schemes under different inflow levels and satisfaction degrees. In addition, the uncertainty analysis of system benefit and water diversion under different unit diversion costs and various inflow levels indicates that the developed IIWRD model is applicable for reflecting uncertainties and solving problems of water distribution of a reservoir in water resources management system.

\section{Data Availability}

The original data used to support the findings of this study are included within the article. 


\section{Conflicts of Interest}

The authors declare that they have no known conflicts of interest or personal relationships that could have appeared to affect the work represented in this paper.

\section{Acknowledgments}

This research was supported by the National Natural Science Foundation of China (no. 51409077), General Program of Natural Science Foundation of Hebei Province (no. D2019402235), Science and Technology Research and Development project of Handan City (no. 1434201078-2), and Scientific Research Project of Hebei University of Engineering (no. 201901400356).

\section{References}

[1] F. Kuria and R. Vogel, "Uncertainty analysis for water supply reservoir yields," Journal of Hydrology, vol. 529, pp. 257-264, 2015.

[2] Q. M. Liang and J. J. Pan, "Analysis of the water supply scheduling in Yuecheng reservoir," Water Conservancy Science and Technology and Economy, vol. 16, no. 11, pp. 1206-1207, 2010.

[3] L. Divakar, M. S. Babel, S. R. Perret, and A. Das Gupta, "Optimal allocation of bulk water supplies to competing use sectors based on economic criterion-an application to the Chao Phraya river basin," Journal of Hydrology, vol. 401, no. 12, pp. 22-35, 2011.

[4] Y. Han, Y. F. Huang, S. F. Jia, and J. H. Liu, "An intervalparameter fuzzy linear programming with stochastic vertics model for water resources management under uncertainty," Mathematical Problems in Engineering, vol. 2013, Article ID 942343, 12 pages, 2013.

[5] Y. Zhou, Y. P. Li, G. H. Huang, and Y. Huang, "Development of optimal water-resources management strategies for kaidukongque watershed under multiple uncertainties," Mathematical Problems in Engineering, vol. 2013, Article ID 892321, 14 pages, 2013.

[6] M. Kang and S. Park, "Modeling water flows in a serial irrigation reservoir system considering irrigation return flows and reservoir operations," Agricultural Water Management, vol. 143, pp. 131-141, 2014.

[7] T. Fowe, H. Karambiri, J.-E. Paturel, J.-C. Poussin, and P. Cecchi, "Water balance of small reservoirs in the Volta basin: a case study of Boura reservoir in Burkina Faso," Agricultural Water Management, vol. 152, pp. 99-109, 2015.

[8] Y. Xu and G. H. Huang, "A risk-based interval two-stage programming model for agricultural system management under uncertainty," Mathematical Problems in Engineering, vol. 2016, Article ID 7438913, 13 pages, 2016.

[9] H. Zhang, M. H. Ha, H. Y. Zhao, and J. W. Song, "Inexact multistage stochastic chance constrained programming model for water resources management under uncertainties," Mathematical Problems in Engineering, vol. 2017, Article ID 1680813, 14 pages, 2017.

[10] A. Seifi and K. W. Hipel, "Interior-point method for reservoir operation with stochastic inflows," Journal of Water Resources Planning and Management, vol. 127, no. 1, pp. 48-57, 2001.

[11] Y. P. Li and G. H. Huang, "Interval-parameter two-stage stochastic nonlinear programming for water resources management under uncertainty," Water Resources Management, vol. 22, no. 6, pp. 681-698, 2008.

[12] X. S. Qin and Y. Xu, "Analyzing urban water supply through an acceptability-index-based interval approach," Advances in Water Resources, vol. 34, no. 7, pp. 873-886, 2011.

[13] X. Guo, T. Hu, T. Zhang, and Y. Lv, "Bilevel model for multireservoir operating policy in inter-basin water transfer-supply project," Journal of Hydrology, vol. 424-425, pp. 252-263, 2012.

[14] Y. Wu and J. Chen, "Estimating irrigation water demand using an improved method and optimizing reservoir operation for water supply and hydropower generation: a case study of the Xinfengjiang reservoir in southern China," Agricultural Water Management, vol. 116, pp. 110-121, 2013.

[15] E. S. Matrosov, I. Huskova, J. R. Kasprzyk, J. J. Harou, C. Lambert, and P. M. Reed, "Many-objective optimization and visual analytics reveal key trade-offs for London's water supply," Journal of Hydrology, vol. 531, pp. 1040-1053, 2015.

[16] M. Q. Suo, P. F. Wu, and B. Zhou, "An integrated method for interval multi-objective planning of a water resource system in the eastern part of Handan," Water, vol. 9, no. 7, pp. 1-17, 2017.

[17] F.-J. Chang and K.-W. Wang, "A systematical water allocation scheme for drought mitigation," Journal of Hydrology, vol. 507, pp. 124-133, 2013.

[18] H. Y. Guo, H. H. Shi, and X. S. Wang, "Dependent-chance goal programming for water resources management under uncertainty," Scientific Programming, vol. 2016, Article ID 1747425, 7 pages, 2016.

[19] J. Z. Salazar, P. M. Reed, J. D. Quinn, M. Giuliani, and A. Castelletti, "Balancing exploration, uncertainty and computational demands in many objective reservoir optimization," Advances in Water Resources, vol. 109, pp. 196-210, 2017.

[20] Y. P. Li, G. H. Huang, and S. L. Nie, "Mixed interval-fuzzy two-stage integer programming and its application to flooddiversion planning," Engineering Optimization, vol. 39, no. 2, pp. 163-183, 2007.

[21] Y. P. Li, G. H. Huang, Y. F. Huang, and H. D. Zhou, "A multistage fuzzy-stochastic programming model for supporting sustainable water-resources allocation and management," Environmental Modelling \& Software, vol. 24, no. 7, pp. 786-797, 2009.

[22] S. Wang and G. H. Huang, "Interactive two-stage stochastic fuzzy programming for water resources management," Journal of Environmental Management, vol. 92, no. 8, pp. 1986-1995, 2011.

[23] Y. Xu, G. H. Huang, and T. Y. Xu, "Inexact management modeling for urban water supply systems," Journal of Environmental Informatics, vol. 20, no. 1, pp. 34-43, 2012.

[24] Y. Lv, G. Huang, and W. Sun, "A solution to the water resources crisis in wetlands: development of a scenario-based modeling approach with uncertain features," Science of The Total Environment, vol. 442, pp. 515-526, 2013.

[25] P. Guo, G. H. Huang, and Y. P. Li, “An inexact fuzzy-chanceconstrained two-stage mixed-integer linear programming approach for flood diversion planning under multiple uncertainties," Advances in Water Resources, vol. 33, no. 1, pp. 81-91, 2010.

[26] S. Wang and G. H. Huang, "A two-stage mixed-integer fuzzy programming with interval-valued membership functions approach for flood-diversion planning," Journal of Environmental Management, vol. 117, pp. 208-218, 2013.

[27] Y. Wang, W. Zhang, Y. Zhao, H. Peng, and Y. Shi, "Modelling water quality and quantity with the influence of inter-basin 
water diversion projects and cascade reservoirs in the middlelower Hanjiang river," Journal of Hydrology, vol. 541, pp. 1348-1362, 2016.

[28] Y. Li, Q. Cui, C. Li et al., "An improved multi-objective optimization model for supporting reservoir operation of China's south-to-north water diversion project," Science of The Total Environment, vol. 575, pp. 970-981, 2017.

[29] M. Yu, C. Wang, Y. Liu, G. Olsson, and C. Wang, "Sustainability of mega water diversion projects: experience and lessons from China," Science of The Total Environment, vol. 619-620, pp. 721-731, 2018.

[30] S. S. Sana, "A production-inventory model of imperfect quality products in a three-layer supply chain," Decision Support Systems, vol. 50, no. 2, pp. 539-547, 2011.

[31] J.-R. Lin, T.-H. Yang, and Y.-C. Chang, "A hub location inventory model for bicycle sharing system design: formulation and solution," Computers \& Industrial Engineering, vol. 65, no. 1, pp. 77-86, 2013.

[32] W. Zhu and $\mathrm{Z}$. Wu, "The stochastic ordering of mean-preserving transformations and its applications," European Journal of Operational Research, vol. 239, no. 3, pp. 802-809, 2014.

[33] M. Schneider, K. Biel, S. Pfaller, H. Schaede, S. Rinderknecht, and C. H. Glock, "Using inventory models for sizing energy storage systems: an interdisciplinary approach," Journal of Energy Storage, vol. 8, pp. 339-348, 2016.

[34] D. C. Wilson and A. R. Ek, "Imputing plant community classifications for forest inventory plots," Ecological Indicators, vol. 80, pp. 327-336, 2017.

[35] J. M. Maestr, M. I. Fernández, and I. Jurado, "An application of economic model predictive control to inventory management in hospitals," Control Engineering Practice, vol. 71, pp. 120-128, 2018.

[36] J. X. Liu and Z. A. Yang, "Discussion on the water allocation plan of Yuecheng reservoir," Haihe Water Resources, vol. 2, pp. 16-18, 2008, in Chinese.

[37] P. Du, Research on Water Quantity and Quality Management Model of River Basin under Uncertainty, North China Electric Power University, Beijing, China, 2013.

[38] F. Yi and J. Li, "Analysis of increasing water storage in Yuecheng reservoir after reinforcement," Haihe Water Resources, vol. 5, pp. 69-70, 2011, in Chinese.

[39] W. Li, Y. P. Li, C. H. Li, and G. H. Huang, "An inexact twostage water management model for planning agricultural irrigation under uncertainty," Agricultural Water Management, vol. 97, no. 11, pp. 1905-1914, 2010.

[40] M. Q. Suo, Y. P. Li, G. H. Huang, Y. R. Fan, and Z. Li, “An inventory-theory-based inexact multi-stage stochastic programming model for water resources management," Mathematical Problems in Engineering, vol. 2013, Article ID 482095, 15 pages, 2013.

[41] J. Laherrere, "Estimates of oil reserves," in Proceedings of the EMF/IEA/IEW meeting IIASA, Laxenburg, Austria, June 2001.

[42] M. M. Li, Study on the Optimizing Allocation of Anyang City Water Resources, Gansu Agricultural University, Lanzhou, China, 2008.

[43] T. F. Wang, "Construction and accounting for the project of water diversion cost," Guide of Sci-Tech Magazine, vol. 18, p. 304, 2012, in Chinese.

[44] W. K. Ma, "Analysis of the flood dispatching of " 96.8 " in Yuecheng reservoir," Haihe Water Resources, vol. 1, pp. 3335, 2006, in Chinese.

[45] T. F. Wang, "The construction and accounting of the cost of water diversion project," Guide of Science and Technology, vol. 18, p. 304, 2012, in Chinese. 